\title{
Influence of Laser Beam Polarization on Laser Micro-Machining of Molybdenum
}

\author{
Rémi TORRES ${ }^{* 1}$, Thomas KAEMPFE ${ }^{* 2}$, Martin DELAIGUE ${ }^{* 3}$, Olivier PARRIAUX ${ }^{* 2}$, Clemens HÖNNINGER ${ }^{* 3}$, John \\ LOPEZ $^{* 1,4}$, Rainer KLING ${ }^{* 1}$, Eric MOTTAY*3 \\ ${ }^{* 1}$ ALPhANOV, 351 cours de la liberation, batîment, A11, 33405 Talence, France \\ E-mail: remi.torres@alphanov.com \\ ${ }^{*}$ Université de Lyon, Laboratoire H. Curien UMR CNRS 5516, F-42000 Saint Etienne, France \\ *3 Amplitude Systemes, 11 avenue de Canteranne, Cité de la Photonique, 33600 Pessac, France \\ ${ }^{*}$ Université de Bordeaux 1, Celia UMR 5107 cours de la Libération, 33405 Talence, France
}

\begin{abstract}
Ultrashort pulse lasers have been established as precise and universal tools for the micromachining of solid materials (cutting, texturing ...). For these applications the quality of the cutting cross-section is important. The use of a Gaussian beam profile and linear polarization leads to tapered cutting sidewalls. It is possible to change the polarization orientation in order to machine a material for obtaining straight and vertical sidewalls. For this purpose a specific polarization converter was used. The transformation of the polarization distribution from linear to radial and azimuthal is done by a subwavelength, binary grating creating a $\pi$ phase shift between the TE and TM transmitted waves. In this paper we report on investigations on the influence of laser polarization (radial, azimuthal, circular, linear) on the ablation characteristics of molybdenum and PZT using an $\mathrm{Yb}$ doped crystal laser (500 fs) and compare these results with previously published results.

DOI: $10.2961 / \mathrm{j} 1 \mathrm{mn} .2013 .03 .0001$
\end{abstract}

Keywords: Azimuthal polarization, radial polarization, micro-machining, texturation, etch rate, femtosecond, micro machining

\section{Introduction}

It is now recognized that the ultrashort pulse laser machining is very high quality. It minimizes the thermal effects and provides a high cutting edge quality [1]. However, the laser beam's energy distribution is a gaussian. It causes a conicity of cutting edge. A decisive factor for the geometry of the cutting edge is the polarization [2]. The absorption of the energy of the laser pulse is a function of this parameter. The laser source emits a generally linearly polarized beam. So, the edge conicity depend of the laser polarization. For this study, we used a $0^{\text {th }}$ order grating polarization converter made by the $\mathrm{H}$. Curien Laboratory. The state of the art setup in the form of a corrugation etched directly into a high index substrate needs very small periods $(<\lambda / 4)$ and a very large height-to-width ratio (»5) of the ridge, and shows significant losses due to reflection $(>10 \%$ for TE). In the present paper a grating etched into a high index layer situated on top of a low index substrate is used. The optimized modal behavior of such a setup allows using more favorable grating parameters (larger period, aspect ratio of about 2) and enables a close to $100 \%$ transmission for both polarization components [3]. Hydrogenated amorphous silicon (a-Si:H, n=3.7) on a fused silica substrate is used as the high index layer material. For the grating fabrication, a Cr-mask made by ebeam lithography is transferred by contact UV-lithography into a resist layer, which is subsequently used to etch the grating into the a-Si:H layer by reactive ion beam etching [4]. Furthermore, there is a potential for mass fabrication by resorting to the batch planar micro- structuring technologies of microelectronics.

Our paper is organized as follows: we first describe the experimental setup, as well as the experimental protocol, then we describe the result and we will finish by describing potential application.

\subsection{Experimental setup}

Figure 1 illustrates the experimental setup. We use two femtosecond lasers with a linear polarization. These are Yb-doped crystal femtosecond lasers from Amplitude Systemes. The first model is a S-Pulse HP. The pulse duration is $500 \mathrm{fs}$. The maximum pulse energy is $100 \mu \mathrm{J}$ the maximum repetition rate $10 \mathrm{kHz}$ and the average power $1 \mathrm{~W}$. The second laser is a S-Pulse HP2: pulse duration 500 fs, maximum pulse energy $2 \mathrm{~mJ}$, maximum repetition rate $300 \mathrm{kHz}$ and average power $8 \mathrm{~W}$. We use an energy controller device based on a polarization filter and a beam expander. For changing the beam polarization we use a converter (radial and azimuthal polarization) and a quarter wave plate (circular polarization). The beam is injected in a 2-axis galvo head. A lens focuses the beam onto the sample $(\mathrm{f}=100 \mathrm{~mm})$ and a set of XYZ motorized stages is used for positioning the sample under the beam. The beam diameter $\left(1 / \mathrm{e}^{2}\right)$ is measured in the focus plane by a commercial beam analyzer (WinCam); the spot diameter is $40 \mu \mathrm{m}$. An aspiration device is used to collect gas and dust produced during laser ablation. 


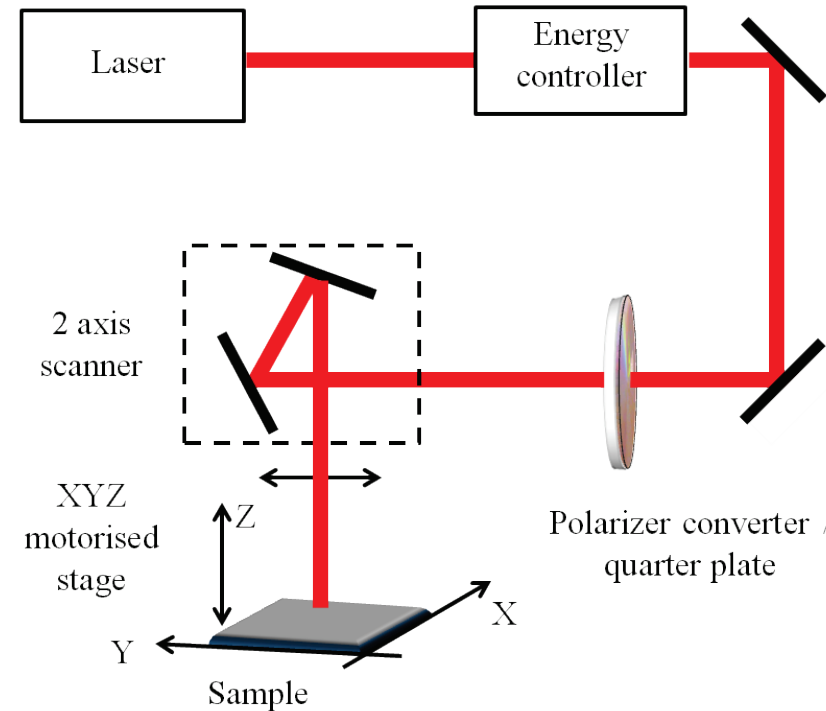

Fig. 1 Experimental Setup.

\subsection{Experimental protocol}

Four polarization states have been used for this work: azimuthal, radial, circular, linear. For obtaining these polarizations we used several techniques. For generating the radial and azimuthal polarizations, we use a polarization converter. A converter can provide both polarizations: one orientation provides the azimuthal polarization, the other, at 90 degrees, provided the radial polarization. Figure 2 illustrates the operation of the converter.

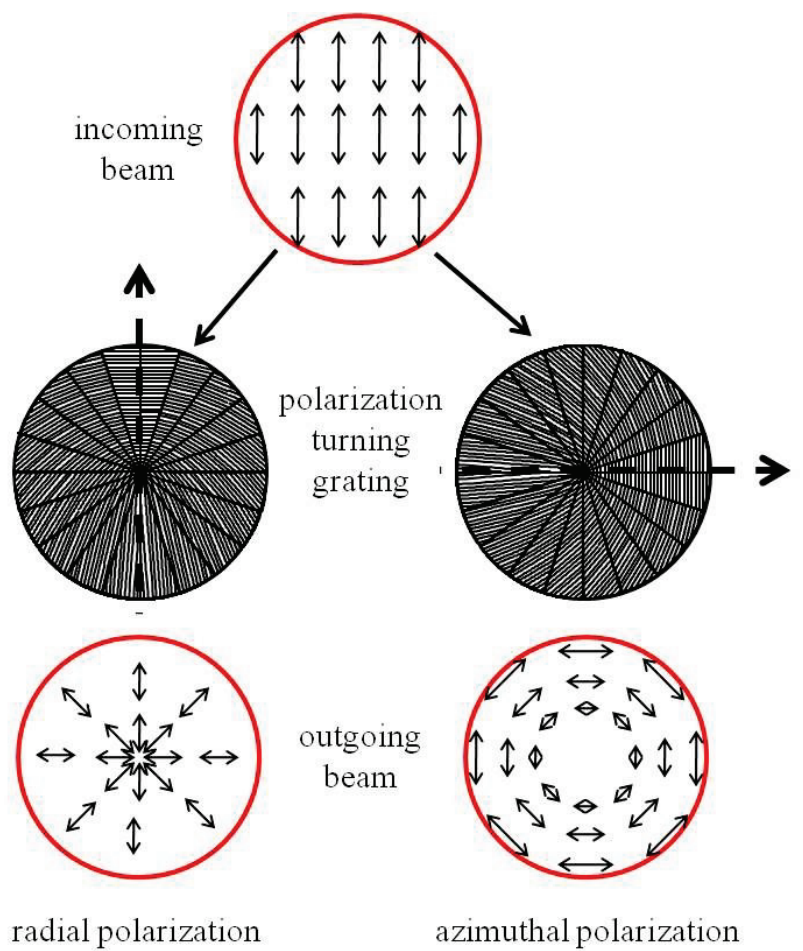

Fig. 2 Orientation of the polarization converter
For the circular polarization, we used a quarter-wave plate with a linearly polarized input. For the linear polarization we used any convenient element. The pulse energy is measured with a power meter before the entrance of the galvo head. The target samples are $100 \mu \mathrm{m}$-thick molybdenum foils. The experimental protocol is based on a single pass process using a Gaussian beam profile. For each polarization state and each pulse energy level, we have produced a pattern of parallel lines, where each line is made using a different scan velocity, ranging from 0.1 $\mathrm{mm} / \mathrm{s}$ to $25 \mathrm{~mm} / \mathrm{s}$. The length of each line is long enough $(10 \mathrm{~mm})$ in order to have a stable and constant scan-speed in the middle of the line, even at high speed. The width, the depth and conicity of the groove are measured using a laser scanning confocal microscope (figure 3). This microscope has a resolution of $0.16 \mu \mathrm{m}$ in the $\mathrm{X}, \mathrm{Y}$ and $<3 \mathrm{~nm}$ in $\mathrm{Z}$. The processing quality is investigated by means of an SEM. We have only considered scan velocities that give no "over thickness inside the groove and for which the groove is deep enough for a significant depth-measurement. Since the groove has a triangular shape, we can easily calculate the volume of the groove and then calculate the etch rate. Thus, each average power value in the graph is associated to several etch rate values. Such a protocol enables us to determine the more relevant scan velocity-window (operating points) for each pulse energy value. Finally, we have only keep the upper part of the curve and we will call them the best operating points [5].
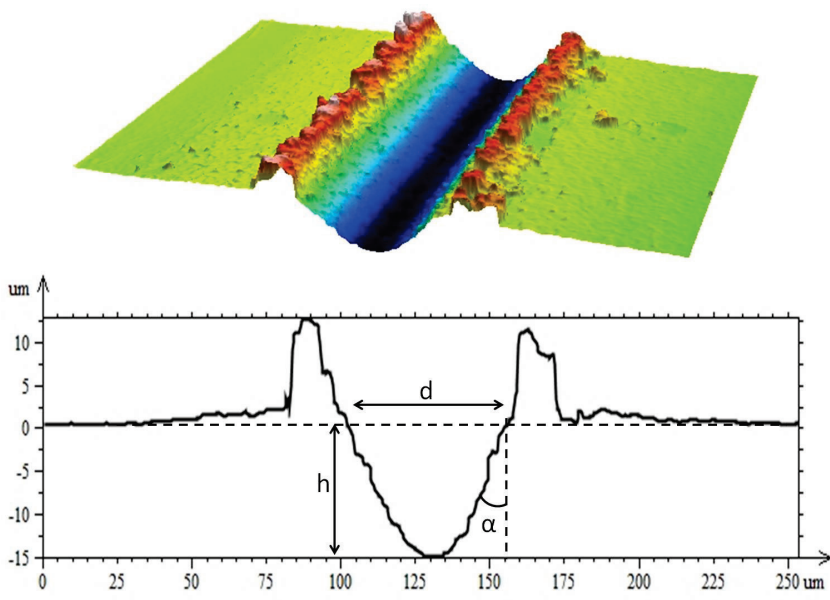

Fig. 3 Groove processed with pulse energy of 23,5 $\mu \mathrm{J}, 10 \mathrm{kHz}$ rep rate and $1 \mathrm{~mm} / \mathrm{sec}$ scan speed. Width (d), depth_(h) and conicity $(\alpha)$ measured using a laser scanning confocal microscope. $3 \mathrm{D}$ view (top) and profile measurement (bottom)

\section{Results}

In laser cutting, light absorption is the most important physical parameter. So, the absorption depends of the angle between the side wall and beam direction and the orientation of the polarization relative to the plane of incidence [6-7]. In this perspective, it becomes clear that the polarization of the laser beam used plays a major role. In laser cutting of metals it has been found that the most effective is the radial polarization and the main reason was identified [8]. The energy deposition on the edge must be 
maximum, therefore the polarization must be perpendicular to the side wall, which is the case for the radial polarization. A radially polarized beam is always p-polarized relative to the cutting front and the side wall, while an azimuthally polarized beam is s-polarized and will be more suitable for drilling [9]. The circular polarization is a mixture of both $p$ and s linear polarizations, it turns as function the time. On Figure 4, we have plotted the conicity as a function the depth for each groove produced with the previous experimental protocol. The angle is calculated at a precision of ranging 0.5 to $1 \%$. We have chosen only the best operating points. That is to say for the same depth obtained with different experimental parameters (P, f, V), we chose the smallest angle. It appears clearly that a radial polarization causes a lower conicity than other polarizations. For a constant depth $(23 \mu \mathrm{m})$, we obtain $13.3^{\circ}$ about the conicity. This is due to the distribution of the absorption of laser energy in the material. With radial polarization (p-polarization) we increase the light absorption on the side wall of groove. So, it improves the ablation in this area.

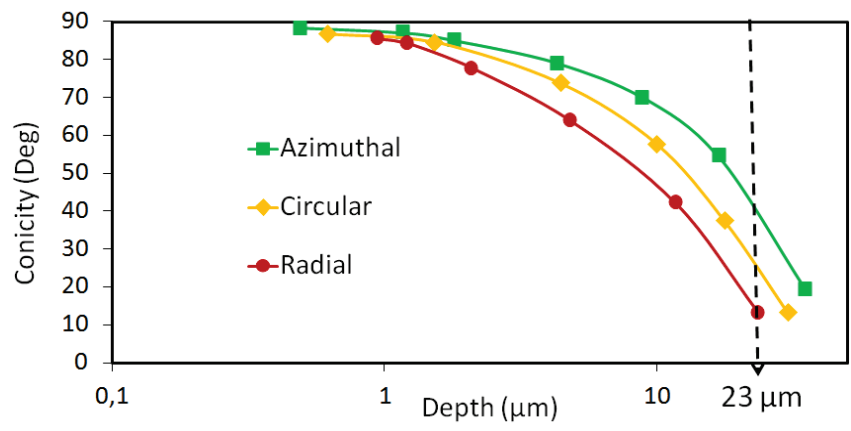

Fig. 4 Conicity as a function of the average power

We have applied this process at a PZT sample to cut all over the material. We have chosen this material for this application like piezoelectric in aeronautic and because it's more thick than molybdenum (thickness is $210 \mu \mathrm{m}$ ). We used a multi-pass process at $\mathrm{f}=40 \mathrm{kHz}, \mathrm{E}=47,5 \mu \mathrm{J}$. The table 1 summarizes the results for different kind of polarization.

We obtain the best result for linear $\mathrm{p}$ with $3^{\circ}$ about the tapered. It's because in this case the polarization is oriented

Table 2 Conicity as function the polarization

\begin{tabular}{cc}
\hline Polarization & Conicity $($ Deg) \\
\hline Radial & $3.9 \pm 0.04$ \\
Azimuthal & $4.7 \pm 0.047$ \\
Circular & $4.4 \pm 0.045$ \\
Linear $\mathrm{p}$ & $3 \pm 0.03$ \\
Linear $\mathrm{s}$ & $5.8 \pm 0.058$ \\
\hline
\end{tabular}

in one direction and is perpendicular at the side wall of groove. The energy is absorbed on this area. In micromachining, we want to cut a complex pattern and we want to keep the same conicity whatever the scan direction. With a linear polarization the conicity ranged from $3^{\circ}$ (linear $\mathrm{p}$ ) to $5.8^{\circ}$ (linear $\mathrm{s}$ ) as function the scan direction.
Commonly, we used circular polarization to homogenize the polarization effect, the conicity is $4.4^{\circ}$. With radial polarization, we obtained the best compromise: a symmetric profile whatever the scan direction and a good conicity $\left(3.9^{\circ}\right)$.

The polarization of the beam also influences the etch rate and efficiency of ablation. Changing the orientation of the polarization can also change the ablation distribution. With radial polarization the ablation occurs on the cutting front and cutting edge. With azimuthal polarization it occurs in depth. From the information provided by the profile of the grooves (Figure 3) we can calculate the ablation rate. It is given by the following formula :

Etch_rate $=\frac{\mathrm{S} \times \mathrm{V}}{60}$

$\mathrm{S}\left(\mathrm{mm}^{2}\right)$ is the surface of the line section and $\mathrm{V}\left(\mathrm{mm} \cdot \mathrm{s}^{-1}\right)$ the scan velocity. The efficiency $\left[\mathrm{mm}^{3} \cdot \mathrm{min}^{-1} \cdot \mathrm{W}^{-1}\right]$ is obtained by dividing the previous expression by the average power. The results are summarized in Table 2 . With a circular polarization the result is in accordance with literature [10-13]. We observe that by changing the orientation of the polarization we are improving the efficiency of ablation. We obtain a best result with the radial polarization followed by the azimuthal polarization, respectively 0.19 and $0.16 \mathrm{~mm}^{3} \cdot \mathrm{min}^{-1} \cdot \mathrm{W}^{-1}$.

For the reason expressed before distribution of energy on the cutting front improve the efficiency of ablation.

Table 1 Maximum efficiency in power at $10 \mathrm{kHz}$ for different polarization

\begin{tabular}{ccc}
\hline Material & Polarization & Efficiency $\left(\mathrm{mm}^{3} \cdot \mathrm{min}^{-1} \cdot \mathrm{W}^{-1}\right)$ \\
\hline \multirow{3}{*}{ Mo } & Radial & 0.19 \\
& Azimuthal & 0.16 \\
& Circular & 0.10 \\
\hline
\end{tabular}

\section{Application}

The use of radial or azimuthal polarization in the literature has so far been mainly envisaged in micromachining applications (cutting and drilling). In this paper we present another type of application associated with surface texturing. The surface texturing can change the optical property of a surface. It is possible, by laser, to create periodic nanostructure called "ripples" [14]. The effect of diffracting structures is used to color the metal surface [15-17]. Ripples are elongated nanostructures always perpendicular to the laser polarization. The work presented in the literature refers to ripples generated by a linearly polarized beam. Here we present nanostructured surfaces written by radially, azimuthally and linearly polarized beams on molybdenum. We engrave several lines with no overlap between each. We used a single pass process: $\mathrm{V}=50 \mathrm{~mm} / \mathrm{s} ; \mathrm{E}=10 \mu \mathrm{J} ; \mathrm{F}=100 \mathrm{kHz}$. We did this process for each polarization.

In Figure 5, SEM images show the structured area. We find that the ripple orientation vary across the laser spot. 
For the radial polarization, the ripple pattern is an arc at the center of the line and is straight at the edges. For the azimuthal polarization ripples are straight at the center of the line and curved from the center to the edges. For the linear polarization, ripples are parallel to the horizontal direction. We observe those patterns because ripples are perpendicular to the polarization. Figure 6 shows the macroscopic effect. We notice the diffractive effect due to the ripples. We have a brighter effect with radial and azimuthal polarization. It's because there several ripples orientation in a same line compare to linear polarization.
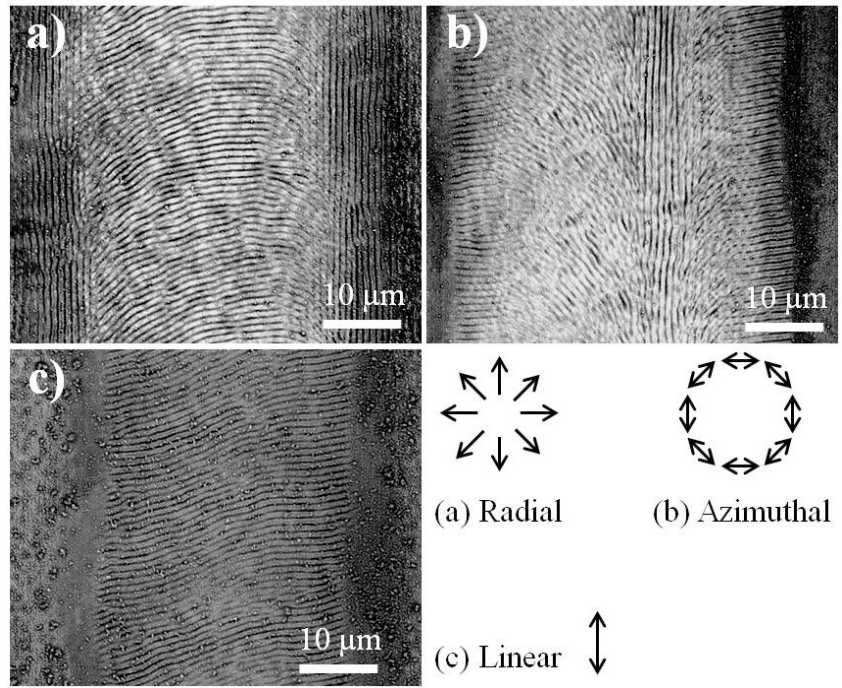

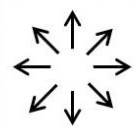

(a) Radial

(c) Linear $\downarrow$
Fig. 5 SEM image of structured surface with several polarizations a) radial, b) azimuthal, c) linear

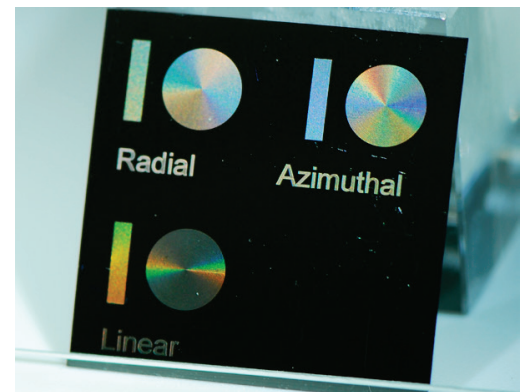

Fig. 6 Macroscopic effect of ripples on molybdenum surface

\section{Conclusion}

The objective of this work was to demonstrate the ability of our polarization converter to improve laser machining on Molybdenum. A comparative study between radial, azimuthal, circular and linear polarizations was conducted. We have shown that the radial polarization is more efficient to engrave and cut material. We can decrease the conicity and improve the ablation efficiency. Future studies will be done to improve deep drilling using azimuthal polarization. We conclude by a potential application to surface texturing. We can create ripples with different orientation in same line and increase the brighter effect on the surface.

\section{Acknowledgments}

(1) We acknowledge the support and funding of the European Commission, the French Ministry of Research and the Aquitaine Regional Council.

(2) We thank A. Zoubir for his participation in consideration of this article.

\section{References}

[1] Y. Bellouard, Champion, A., Lenssen, B., Matteucci, M., Schaap, A., Beresna, M., ... \& Lopez, J. (2012). The femtoprint project. Journal of Laser Micro/Nanoengineering (JLMN), 7(1), 1-10.

[2] Hnatovsky, C., Shvedov, V. G., Shostka, N., Rode, A. V., \& Krolikowski, W. (2012). Polarization-dependent ablation of silicon using tightly focused femtosecond laser vortex pulses. Optics Letters, 37(2), 226-228.

[3] T. Kämpfe and O. Parriaux: J. Opt. Soc. Am. A, 28, (2011) 2235

[4] T. Kämpfe, S. Tonchev, G. Gomard, C. Seassal and O. Parriaux: Photonics Journal, IEEE, 3, (2011) 1142.

[5] J. Lopez, R. Kling, R. Torres, A. Lidolff, M. Delaigue, S. Richaud, C. Hönninger and E. Mottay, Proc. SPIE (2012), Vol 8243

[6] M. Meier, V. Romano and T. Feurer: Applied Physics A, 86, (2006) 329

[7] Nolte, S., Momma, C., Kamlage, G., Ostendorf, A., Fallnich, C., Alvensleben, F. V., \& Welling, H. (1999). Polarization effects in ultrashort-pulse laser drilling Nolte Appl. Phys. A. Applied Physics A, 68, 563-567.

[8] V.G. Niziev, A.V. Nesterov: J.Phys.D, 32, (1999)1455

[9] M. Kraus, M. A. Ahmed, A. Michalowski, A. Voss, R. Weber and T. Graf: Optics express, 18, (2010) 22305

[10]A. Ancona, S. Döring, C. Jauregui, F. Röser, J. Limpert, S. Nolte, and A. Tünnermann : Opt. Lett. 34, (2009) 3304

[11] M. Schmid, B. Neuenschwander, V. Romano, B. Jaeggi and U. Hunziker: Proc of SPIE / Photonics West conference, (2011) p. 91.

[12]B. Jaeggi, B. Neuenschwander, M. Schmid, M. Muralt, J. Zuercher and U. Hunziker, Proc. 6th International WLT conference on Lasers in Manufacturing (LiM), (2011) p. 164

[13] J. Lopez, A. Lidolff, M. Delaigue, C. Hönninger and E. Mottay: Proc. Symp A Quarterly Journal In Modern Foreign Literatures, (2011) p. 1.

[14] Klug, U., Düsing, J. F., Sato, T., Washio, K. and Kling, R.: Proc. SPIE, (2010) Vol 2010, p. 759006

[15] A. Y. Vorobyev, and C. Guo : Appl. Phys. Lett. 92, (2008) 041914

[16] H. Lochbihler: Opt. Express 17, (2009) 12189

[17]B. Dusser, Z. Sagan, H. Soder, N. Faure, J.P. Colombier, M. Jourlin and E. Audouard: Optics express, 18, (2010) 291

(Received: July 4, 2012, Accepted: August 23, 2013) 